\title{
Ciclo hidrosocial y acceso al agua en la periferia de la ciudad de Morelia, México: Estudio de caso en La Aldea
}

\author{
Hydrosocial cycle and access to water in the periphery of the \\ city of Morelia, Mexico: Case study in La Aldea settlement
}

\author{
Lorena García-Estrada ${ }^{1}$ \\ Juan Hernández-Guerrero ${ }^{2}$ \\ Universidad Autónoma de Querétaro, México
}

\begin{abstract}
Resumen
El agua es indispensable en el metabolismo de las ciudades, pero la acelerada expansión acentúa, hacia las periferias, problemas de acceso, distribución y calidad, los cuales suelen ser utilizados por actores con poder socio-político que debilitan el ciclo hidrosocial. El presente trabajo analizó el acceso al agua a través del ciclo hidrosocial en la periferia norte de la ciudad de Morelia, México, específicamente, en el asentamiento La Aldea. El objetivo se contestó con recorridos de campo, observaciones, entrevistas y encuestas. Los resultados indican estrés en el ciclo hidrosocial, donde el flujo es disímil y supeditado por movimientos políticos y desarrolladoras. Además, disminuyó la participación de la población e incrementó la compra de agua embotellada y camiones cisterna, mientras que la intervención de las autoridades es limitada y permisible.
\end{abstract}

Palabras clave: ciclo hidrosocial, acceso al agua, periferia urbana, actores de poder.

\begin{abstract}
Water is indispensable in the metabolism of cities, but the accelerated peripheral expansion of cities accentuates problems of water access, distribution and quality, which, in turn, are often used by actors with socio-political power that weaken the hydrosocial cycle. The present study analyzed access to water through the hydrosocial cycle in the northern periphery of the city of Morelia, Mexico, specifically in the settelement of La Aldea. The study's objective was met with field trips,

1 Maestra en Geografía. Universidad Autónoma de Querétaro. Facultad de Ciencias Naturales. Campus Aeropuerto. Carretera a Chichimequillas s/n, Ejido Bolaños. C.P. 76140. Correo electrónico: mariana. lorena.ge@gmail.com

2 Doctor en Geografía. Universidad Autónoma de Querétaro. Facultad de Ciencias Naturales. Campus Aeropuerto. Carretera a Chichimequillas s/n, Ejido Bolaños. C.P. 76140. Correo electrónico: juan.hernandez@uaq.mx
\end{abstract}


observations, interviews and surveys. Results indicate stress in the hydrosocial cycle wherein there is a dissimilar flow subordinated by political movements and infrastructure development groups. Additionally, population involvement decreased, bottled water and water trucks purchases increased, all while the intervention of the authorities was limited and permissible.

Keywords: Hydrosocial cycle; Water access; Urban periphery; Power actors.

\section{Introducción}

La accesibilidad al agua forma parte de los derechos humanos desde el año 2010 y uno de los principales temas y retos por atender en la agenda del siglo XXI (Naciones Unidas, 2010). En la Meta 7.C de los Objetivos de Desarrollo del Milenio se menciona el avance en la reducción del porcentaje de personas sin acceso al agua, pasó de $76 \%$ en 1990 a $91 \%$ en 2015, ello equivale a 1900 millones de personas con acceso seguro al agua, pero todavía 663 millones no lo tienen (Naciones Unidas, 2015). El compromiso continúa en el sexto objetivo de la Agenda 2030 para el Desarrollo Sostenible, donde la meta 6.1 señala el acceso universal y equitativo al agua potable (Gobierno de México, 2018).

Lo anterior es un reto importante en ciudades de acelerado crecimiento, las cuales al tratar de ser competitivas experimentan fenómenos de atracción y concentración (poblacionales y económicos) con cada vez más necesidades, entre ellas el agua, problema que se acentúa conforme su expansión, donde la rápida ocupación y consolidación de las periferias urbanas promueve una intensa transformación territorial que dificulta el acceso a servicios e infraestructura (Castro y Rodrigues, 2017; Kaushal et al., 2015; Narain, 2016). Además, el acceso al agua en las periferias urbanas suele involucrar tensiones asociadas con actores de poder que acompañan la propia dinámica de esos sectores; escasa vigilancia, fragmentación, susceptibles al cambio, difícil acceso, limitación de servicios y empoderamiento particular de fuentes hídricas de abastecimiento (Aguilar, 2008; Karpouzoglou et al., 2018; Metha et al., 2014; Díaz-Caravantes y Wilder, 2014).

Ante las diferentes aristas del acceso al agua en las periferias urbanas, los análisis deben de ir más allá de un enfoque hidrológico (eje estratégico y articulador), pues se debe incluir el contexto político, económico y cultural, ya que la manipulación y control del agua contiene actores sociales e instituciones (Budds, 2008). Por lo mismo, definir o re-definir estrategias y esfuerzos para acceder al agua por diferentes actores, incluye tensiones y 
conflictos con grupos de poder en complacencia con autoridades (permisibles, corruptas o desinteresadas), ello abre la brecha de procesos negativos híbridos socio-naturales para acceder al agua; fuentes de abastecimiento, distancia, tiempo, confiabilidad y costos (Meerganz von Medeazza, 2006; Linton y Budds, 2014; Delgado-Ramos, 2015).

El estudio de esos problemas se ha tratado desde el enfoque del ciclo hidrosocial, el cual hibridiza lo socio-natural, y suele apoyarse de dos posturas. La primera desde la filosofía política, donde el acceso al agua es resultado de la interacción entre gestión del agua, normas sociales y legales, el estado y el ambiente (Budds, 2012; Schmidt, 2014; Arahuetes et al., 2016). La segunda es desde la ecología política urbana, donde se enfatiza el flujo de agua y las relaciones de poder para demostrar la naturaleza politizada de su gestión (Swyngedouw, 2009; Budds et al., 2014; Linton y Budds, 2014). Ambos enfoques permiten entender las problemáticas de su acceso, pero el segundo al tener el carácter urbano posibilita mejores argumentos para el tema aquí planteado, ya que se requiere de la comprensión de las relaciones de poder y sus interacciones políticas, socioeconómicas y ecológicas en esos espacios a multiescala y en diferente tiempo y espacio (Swyngedouw et al., 2002; Schmidt, 2014; Arahuetes et al., 2016; Olvera-Molina, 2016).

Algunos estudios con enfoque hidrosocial realizados en ciudades de Ecuador, Chile, España y Grecia explicaron que los problemas de inequidad en el acceso al agua están supeditados por relaciones de poder que, junto con el inadecuado uso, manejo y gestión del agua, forman subsistemas de control con mecanismos de beneficio individual, y que en algunos casos tienen que realizar nuevos y costosos procesos para su obtención y distribución, o bien, involucrarse con grupos de poder (Swyngedouw, 1997; Kallis, 2010; Budds, 2012; Arahuetes et al., 2016). Por lo mismo, en periferias urbanas el tema debe abordarse con mayor detalle, debido que los conflictos se observan en pequeños mosaicos o fragmentos con problemas diferenciados entre sí en complacencia con las autoridades.

El caso de las ciudades mexicanas y sus periferias no es ajeno a lo señalado, si bien, los estudios bajo el enfoque hidrosocial no son tan bastos, los existentes han permitido comprender la complejidad de los flujos hídricos y la manipulación de su naturaleza con fines desarrollistas y oportunistas, así como de dominación y opresión (Olvera-Molina, 2016). Se distinguen estudios que han visibilizado el problema en algunas ciudades y sus periferias. 
López-Mares et al. (2019) analizaron las transformaciones sociales y paisajísticas en ríos de la ciudad de San Luis Potosí y concluyeron con una fragmentación física y simbólica. Vilchis-Mata et al. (2018) evaluaron las relaciones entre disponibilidad, demanda, adaptación y gestión de agua en la ciudad de Toluca, y determinaron que la sostenibilidad y disponibilidad del agua está en riesgo por malos manejos del Estado. Rodríguez (2018) estudió la apropiación del agua y relaciones de poder en Los Altos de Jalisco y concluye que los problemas se deben al deficiente manejo hídrico nacional y privilegios regionales. Aunado a esos trabajos, existen estudios que sin ser denominados hidrosociales también hibridizan los flujos de agua, y en todos los casos resalta el deficiente acceso al agua como proceso paralelo a situaciones de especulación de suelo, sobreexplotación de agua superficial y subterránea, corrupción, venta clandestina, irregularidades con desarrollos inmobiliarios y desperdicio de agua (Domínguez, 2006; Ávila, 2007; Delgado-Ramos, 2015; Tetreault y McCulligh, 2018).

Problemas similares se acentúan o recién se forman en ciudades del centro del país, especialmente aquellas mayores a 500 mil habitantes (Ciudad de México, Guadalajara, Puebla, Toluca, León, Querétaro, San Luis Potosí, Aguascalientes, Cuernavaca, Morelia, Celaya, Pachuca, Tlaxcala), las cuales presentan altas tasas de crecimiento ( $2 \%$ superiores al $1.8 \%$ del país), suman 40 millones de personas, presentan 22 acuíferos sobreexplotados, uso consuntivo hasta $500 \mathrm{hm}^{3}$, cobertura de agua potable del $70 \%$, construcción de grandes obras de ingeniería en sitios distantes (ej. Acueducto II) y constantes conflictos socio-políticos (Ávila, 2007; Vieyra et al., 2018; López, 2013; SEMARNAT y CONAGUA, 2015; Olvera-Molina, 2016; SEDATU y CONAPO, 2018; López-Mares et al., 2019).

De los casos antes citados, el presente trabajo aborda la ciudad de Morelia, la cual históricamente, y a pesar de contar con fuentes de agua que cubren la demanda, presenta problemáticas de acceso al agua de índole social, económico y político; contaminación, acceso diferenciado, transitoriedad del servicio, manipulación, deficiente infraestructura, intereses dominantes y fuerzas de poder sobre el agua (Morales, 2015; Ávila, 2007).

Los problemas trascendieron a diferentes sectores de la ciudad, siendo la periferia norte una de las más afectadas, específicamente la zona conocida como La Aldea. En ella se localizan pozos antiguos y sobreexplotados, donde se extrae agua del flujo intermedio a la profundidad del 
manto freático, entre 150m y 160m (Garduño et al., 2014). Además, ese sector está involucrado en deficientes procesos de gestión y manejo del suelo, lo que ha generado que el principal sistema operador de agua potable y alcantarillado no tenga todos los argumentos legales para brindar el servicio de agua, aunque sí se conoce de su participación en algunos implementos de infraestructura, apoyo con información y calidad de agua (Ávila, 2007; Morales, 2015). Al respecto, el deficiente acceso y la transitoriedad de la cantidad de agua han generado enfrentamientos entre habitantes, autoridades y actores con privilegios en el acceso y posesión de fuentes de agua. A pesar del problema, se identifican insuficientes datos, diagnósticos y análisis, aun cuando es un polo de desarrollo urbano y el agua es un detonante de fragmentación y reconfiguración territorial.

Con los argumentos señalados surgen las siguientes interrogantes ¿cuáles son las interacciones sociales y políticas que están involucradas en el acceso al agua?, ¿qué implicaciones socioeconómicas se presentan para acceder al agua?, y ¿cuáles son los indicadores (entradas, dirección, consumo y salidas) que condicionan el ciclo hidrosocial? Así, el presente trabajo tiene como objetivo analizar el acceso al agua en la periferia norte de la ciudad de Morelia mediante el ciclo hidrosocial para casos de estudio de La Aldea.

El artículo presenta una estructura de la siguiente forma. Posterior a la introducción, el segundo apartado describe los métodos y herramientas que se utilizaron para colectar y analizar los datos. El tercer apartado contextualiza el proceso socio-político de gestión y administración del agua. En el cuarto apartado se examina el acceso, costo y aprovechamiento del agua por parte de los habitantes de La Aldea. En el quinto apartado se evalúan las variables del ciclo hidrosocial (2012-2018). Finalmente, el documento concluye con un ciclo hidrosocial donde las entradas se relacionan con la manipulación de agua subterránea, compra de agua embotellada y la captación de agua de lluvia, todo asociado a la escasa participación gubernamental, la importante manipulación por grupos de poder y la displicente participación colectiva de la población.

\section{Área de estudio}

La Aldea se ubica al noreste de la ciudad de Morelia, México (a 7 kilómetros del centro urbano) y delimita con el municipio de Tarímbaro (Figura 1). Al año 2010, contaba con 6189 habitantes (INEGI, 2010) 
distribuidos en diez asentamientos, los cuales presentan un grado de marginación alto a muy alto (IMPLAN, 2017). La Aldea surge como un ejido en el año 1936, establecido sobre la zona baja de la subcuenca fluviolacustre de la cuenca del Lago de Cuitzeo, esa cuenca corresponde al Valle de Morelia-Queréndaro, donde se encuentran los acuíferos de Valle de Morelia, Lago de Cuitzeo y Planicie Álvaro Obregón-Queréndaro, siendo este último donde se localiza La Aldea y es considerado el de mayor extensión y espesor de todo el valle (Bravo et al., 2012; Garduño et al., 2014). El clima es templado subhúmedo con temperatura media anual que oscila entre $14^{\circ} \mathrm{C} \mathrm{a} 18^{\circ} \mathrm{C}$ y con una precipitación anual de $700 \mathrm{~mm}$. Actualmente el uso de suelo y vegetación en casi $50 \%$ del lugar es habitacional (420 ha de las 859 ha totales), seguido de $20 \%$ de pastizal y $30 \%$ de parcelas de agricultura de temporal (INEGI, 2010).

Figura 1. Localización de La Aldea


Fuente: Elaboración con base en la información del INEGI (2010) e IMPLAN (2017).

\section{Métodos y herramientas}

Con el fin de responder al objetivo del presente trabajo se emplearon métodos integrados con información de fuentes cualitativas y cuantitativas, así como datos primarios y datos secundarios a través de un proceso constituido por recorridos exploratorios, análisis documental, entrevistas, 
encuestas y observaciones directas. El trabajo de campo se llevó a cabo en los años 2016, 2017 y 2018, específicamente, comprende tres meses de 2016, siete meses de 2017 y dos meses de 2018.

El análisis documental se llevó a cabo mediante publicaciones oficiales disponibles, sean el programa de Desarrollo Urbano de la Zona Oriente de Morelia (Ayuntamiento de Morelia, 2012), Decreto de expropiación de tierras (DOF, 1931) y la Escritura Pública 27992 (IVEM, 2011). También se utilizó información geográfica y estadística digital del INEGI (2010) e IMPLAN (2017) para ser utilizada en un sistema de información geográfica y representar espacialmente los fenómenos en la zona de estudio.

Al mismo tiempo que se realizaba el análisis documental se llevaron a cabo recorridos exploratorios y recorridos guiados por la zona de estudio para lograr un primer acercamiento al lugar y contacto con los habitantes a través de pláticas informales, así como análisis de observación directa y se construyó cartografía participativa con apoyo del guía. En total se realizaron seis recorridos, dos exploratorios y cuatro con un guía, en este segundo caso se recabó información de la cobertura y distribución del servicio de agua, ubicación de las fuentes de abastecimiento, y la geomorfología de la zona, asimismo, se levantaron reportes fotográficos para fundamentar y evidenciar el problema.

Posterior a los recorridos exploratorios y pláticas informales se diseñó y aplicó una encuesta estructurada compuesta de preguntas sobre la continuidad del servicio, cantidad de agua a la vivienda, costo económico en la administración del servicio, uso del agua, fuente de abastecimiento y consumo de agua. Se aplicaron diez encuestas por asentamiento, cantidad establecida por Domènech et al. (2011) debido a la distribución dispersa de los asentamientos. La técnica de aplicación fue uno a uno, es decir, encuestador-encuestado. Se eligieron hombres y mujeres mayores de edad (18 años) que tuvieran conocimiento sobre el servicio y uso del agua. La aplicación se llevó acabo en fines de semana entre 10:00h y 15:00h, esto por seguridad y facilidad de encontrar a los encuestados.

Finalmente, se diseñó y aplicó una entrevista a informantes clave. Las características de los entrevistados fueron, primordialmente, que tuvieran un cargo importante o estuvieran involucrados dentro de la gestión del agua a nivel municipal y local (en cada uno de los asentamientos). Los entrevistados fueron nueve, mismos que se definieron con la técnica de 
bola de nieve; 1) Encargado de Distritos Hidrométricos y Detención de Fugaz del OOAPAS; 2) Encargado de control de Calidad del Agua; 3) Jefa de Estudios y Proyectos del IVEM; 4) Presidente del Comisariado Ejidal de La Aldea; 5) Presidente del Comité de Pozo Uno de La Aldea; 6) Presidente del Comité de Pozo Dos de Ampliación la Aldea; 7) Encargado del Orden Lomas la Aldea; 8) Informante Uno Villas Oriente, y 9) Informante Dos Villas Oriente. La entrevista estuvo compuesta por 15 preguntas divididas en cinco secciones: datos generales, organización, infraestructura, gastos económicos y servicio.

\section{Acceso al agua y conflictos sociales en La Aldea}

Desde su origen (1936) y hasta la década de los setenta, La Aldea presentó cambios significativos de reconfiguración territorial, en gran medida cuando se expropió la tierra y se llevó a cabo el establecimiento de ciudad industrial en 1975, al respecto el Comisariado Ejidal de La Aldea señaló que la oferta de empleos en las fábricas repercutió en la atracción poblacional y transformación de vida de los habitantes originarios. En el año de 1984 se divide el ejido en Cotzio y La Aldea, pero los cambios radicales se presentaron en la década de los noventa, cuando La Aldea fue alcanzada por la expansión de la ciudad de Morelia, proceso que fue acompañado de las iniciativas del Programa de Certificación de Derechos Ejidales y Titulación de Solares (PROCEDE), derivado de la reforma al Artículo 27 de la Constitución Mexicana en 1992, ya que brindó la oportunidad a los ejidatarios de vender las tierras que desde hacía tiempo resultaban difíciles de trabajar (Ávila, 2007).

Derivado de lo anterior, algunos ejidatarios decidieron lotificar sus tierras y venderlas a particulares y al gobierno, ambos casos las utilizaron preferentemente para reventa en la construcción de viviendas. Esa situación fomentó la edificación de asentamientos como Lomas La Aldea en 1996, Mariel en 2005, La Nueva Aldea en 2006 y Villas Oriente en 2012. No obstante, los asentamientos se desarrollaron con inadecuada e insuficiente infraestructura, equipamiento y servicios, siendo el agua uno de los principales, y de manera adicional, en el año 2012 los acuíferos fueron declarados sobreexplotados y se comenzaron a perforar nuevos pozos y abastecer la zona (Ayuntamiento de Morelia, 2012). 
Con los argumentos anteriores, se identifican problemas hidrosociales particulares sobre el acceso al agua, todos ellos con procesos y actores diferentes. En el Pueblo de La Aldea y Ampliación La Aldea, por sus condiciones rurales, la máxima autoridad es el Comisariado Ejidal, y en el servicio de agua su papel es realizar los acuerdos ante la Comisión Nacional del Agua (CONAGUA) para obtener los derechos sobre pozos (extracción de agua), así obtuvieron dos que denominaron I y II. Ambos pozos tienen un comité de agua donde se realiza la gestión, administración y mantenimiento del agua, además, en el Pozo I existen vocales que ayudan a recoger cuotas y fungen como auxiliares.

En casi 50 años desde que surgió el comité de agua del Pozo I, las principales problemáticas son por la asignación clandestina de conexiones de agua por parte del Comisariado Ejidal, donde dicha acción cuenta con poca atención de la opinión de los comités de agua, ello ha propiciado limitada disponibilidad y un servicio deficiente, a la vez que ha causado conflictos sociales, entre los usuarios y los representantes de los comités:

[...]se han presentado inconformidades por parte de algunos habitantes debido que el ejidatario que fraccionó y vendió los terrenos otorgó dinero al comisariado ejidal para que les dieran su toma de agua para poder acceder al servicio, pero nunca se habló con el comité del Pozo I para ver si existían las posibilidades de brindarles el servicio. Presidente del Comité del Pozo I.

Otro problema se presenta en Lomas La Aldea, asentamiento irregular que se formó en 1996 cuando los ejidatarios de esas tierras lotificaron y vendieron, pero al no contar con una tenencia segura del suelo, los servicios públicos se han dispuesto por los pobladores. Así, para resolver la falta de agua se decidió en el 2002 comprar a los ejidatarios un pozo dentro de la zona e iniciaron su administración. En esa actividad se designó a un encargado del orden, quien cobra y administra las cuotas, mientras que otra persona conocida como "bombero" se encarga de la limpieza del pozo y la pila (cisterna de captación de agua) y diariamente suministraba agua a las viviendas. Aun cuando la población ignoraba las implicaciones de la administración de un pozo, se logró una actividad óptima.

Contiguo a Lomas La Aldea se instaló, en el 2005, el asentamiento denominado Mariel (fundado por el movimiento conocido como Antorcha 
Campesina $^{3}$ ) e iniciaron negociaciones para venderles agua, pero una semana después fue evidente la irregularidad del servicio en Lomas La Aldea. En el 2016 el Encargado del Orden de Lomas La Aldea anunció que el agua del pozo se había terminado y lo tuvo que cerrar. Al mismo tiempo se presentan inconformidades e incertidumbre, pues el cierre del pozo se llevó de forma espontánea, pues un día anterior todavía se tenía agua con buena presión en las viviendas. Dos meses después del cierre el OOAPAS perforó un pozo en Mariel y construyó una pila para abastecer de agua a ambos asentamientos, pero solo se suministra en Mariel.

[...]El Encargado del Orden y un grupo de vecinos se unieron a Antorcha Campesina, entonces la población se dividió y el Encargado del Orden dejó de lado su responsabilidad. Cuando se presentaban averías, decía que no había dinero, aun cuando se daba cuota por el servicio. Los antorchistas solo le da agua a aquellos que los apoyan, van a sus marchas y juntas, pero además les cobran por el agua, y nosotros ni si quiera podemos acercarnos al pozo. Habitante de Lomas la Aldea.

Posterior al problema, los pobladores de Lomas La Aldea realizaron contratos con el OOAPAS para las conexiones domiciliarias, pero al año 2019 continúan sin servicio. En la entrevista con el encargado de distritos hidrométricos y detención de fugas del OOAPAS, mencionó que el pozo fue entregado en diciembre del 2017, y está siendo operado por el organismo, aunque la distribución del agua está a cargo de los propios representantes de Mariel y Lomas La Aldea; el OOAPAS se limita a encender el equipo hidráulico y realizar acciones de mantenimiento y registrar el consumo de energía eléctrica.

Ante las dificultades por acceder al agua en Lomas La Aldea se llevó a cabo la perforación de otro pozo. El Encargado del Orden argumentó que Antorcha Campesina fue quien solicitó el recurso a CONAGUA, mismo que asciende a tres millones de pesos, mientras que al municipio de Morelia le correspondió un millón y medio de pesos, ambos fueron otorgados para el equipamiento de ese pozo. Si bien los habitantes de Lomas La

3 Organización política fundada en 1974 con el objetivo de organizar a los sectores más marginados y pobres de la sociedad para lograr mejorar el estado de sus comunidades, luchar por una mejor distribución del ingreso y eliminar las profundas diferencias sociales (Somuano, 2007:41). Por otro lado, esa organización ha destacado como promotora de procesos de extorsión, control y poder que al estar coludida con autoridades y partidos políticos acentúa diferencias, presiones y miedo sobre la sociedad, pero también logra obtener simpatizantes en pro de crecimiento y expansión (Nieto, 2014; Gómez-Valdés y Palerm-Viqueira, 2016). 
Aldea señalan que las malas decisiones del Encargado del Orden, aconsejado por líderes antorchitas, agudizaron el problema, el propio Encargado del Orden se justificó:

Yo junto con otros vecinos nos unimos al movimiento porque por medio de ellos, es más fácil prosperar. Varias veces fui al ayuntamiento a solicitar apoyos y equipamiento, y nunca me hicieron caso; además la gente tampoco me acompañaba. Gracias a Antorcha se pudo conseguir la construcción de un tanque elevado y hacer un cárcamo para tener agua segura en toda la zona. La gente no quiere ir a marcha y asistir a juntas, pero es la única manera de que a uno lo escuchen las autoridades. Encargado del Orden Lomas la Aldea.

Por último, están los casos de La Nueva Aldea (2006) y Villas Oriente (2012), los cuales son conjuntos habitacionales derivados de desarrollos inmobiliarios y apoyos de gobierno. Esos asentamientos, al contrario de los antes mencionados deben cumplir con la normativa legal vigente que asegura el equipamiento y servicios necesarios, pero de igual forma que los anteriores, el servicio de agua es deficientes y limitado.

En el caso de La Nueva Aldea, su construcción inició a principios del 2006 a través de la compra de tierras que realizó el gobierno con ejidatarios, las cuales años después fueron ofrecidas a inmobiliarias. El asentamiento está compuesto por cuatro secciones (I, II, III y IV), la zona I fue designada para uso exclusivo de personas con capacidades diferentes (débiles visuales, discapacidad motriz, entre otras), en la construcción de viviendas también participó el Instituto Municipal de Vivienda (IMUVI), pero aunque cuentan con conexiones de agua en la vivienda el servicio es transitorio. Las secciones II y III quedaron inconclusas, pues la inmobiliaria desapareció de forma espontánea, por lo que está incierto su proceso de regularización, mismo que le otorgaría las bases para acceder a los servicios. Finalmente, la sección IV pertenece al partido político acción nacional. En todas las secciones la falta de agua es un problema, por ello en el año 2007 se llevó a cabo la perforación de un pozo en La Nueva Aldea para abastecer las secciones descritas.

Por otro lado, el asentamiento Villas Oriente, producto de la Inmobiliaria y Constructora Solórzano S.A. de C.V. (desde ahora HERSO, por su nombre comercial) es el mejor equipado y cuyo proceso de urbanización 
se ha llevado conforme a la normativa legal, aunque existen varias inconsistencias en el acceso al agua, especialmente, en cómo se obtuvieron los derechos del pozo del que se abastecen, pues es el mismo pozo que se perforó en La Nueva Aldea, ante esa situación ¿por qué la inmobiliaria brinda un servicio de agua a dos asentamientos que provienen de procesos de urbanización independientes y con circunstancias legales diferentes?

En respuesta, a partir del año 2011 HERSO comenzó a brindar el servicio de agua para ambos asentamientos sin ningún tipo de explicación de carácter legal, pero eso tiene respuesta por un fideicomiso entre la inmobiliaria de La Nueva Aldea, el Institutito de Vivienda del Estado de Michoacán y la Impulsora de Vivienda de Michoacán S.A de C.V, todos cedieron a HERSO el terreno donde se construyó La Nueva Aldea y de forma paralela obtuvo la posesión de ambos asentamientos y también la administración y acceso al agua. Aunque cabe señalar que en el Registro Público de Derechos de Agua aparece como titular la Impulsora de Vivienda de Michoacán S.A de C.V.

En el servicio de agua por parte de HERSO existe un encargado de urbanización quien es el responsable de inspeccionar y brindar adecuadamente el servicio y desarrollar los proyectos requeridos por OOAPAS para la autorización del conjunto habitacional. OOAPAS por su parte es la autoridad encargada de vigilar e inspeccionar que los proyectos relativos al servicio de agua y drenaje se lleven adecuadamente por la inmobiliaria para posteriormente otorgar su aprobación. Asimismo, se cuenta con las juntas de mesa conformadas por representantes de los asentamientos, los cuales apoyan con la vigilancia para que el resto de los habitantes hagan un uso adecuado del agua y también comuniquen las demandas e inconformidades hacia la inmobiliaria. Por último está el "bombero", es el técnico que se encarga de distribuir el agua del pozo hacia las viviendas, así como vigilar que el sistema de distribución se encuentre en óptimas condiciones.

Es evidente que el OOAPAS no brinda el servicio de agua en ninguna de los asentamientos de La Aldea. A esa situación se suman dos argumentos, el primero es porque ha sido incapaz de realizar una adecuada gestión y la población no confía en su servicio, tal como sucede en el Pueblo de la Aldea y Ampliación la Aldea donde los ejidatarios junto con los comités de agua decidieron mantenerse al margen de dicho organismo y ser ellos los responsables del servicio, como lo hacían desde que eran considerados un 
ejido, y esto a su vez tiene el respaldo de la población. La segunda es por la situación legal en la que se encuentran los asentamientos, pues Lomas La Aldea, Villas Oriente y La Nueva Aldea no han concluido su construcción, por ende, aún no se entregan al municipio para realizar la regularización de la tenencia del suelo y que se puedan suministrar los servicios.

También es notoria la diferencia de acceso y disponibilidad de agua en los asentamientos, esto se debe a las diferentes decisiones que se han tomado, aunque a mayor poder e involucramiento el acceso y disponibilidad es mejor, esto se observa en unos cuantos, mientras que el resto de los pobladores tienen menores relaciones de poder e involucramiento, lo que equivale a tener menos acceso y disponibilidad de agua.

Con lo señalado hasta el momento y en relación al trabajo de Arahuetes et al. (2016), se comprobó que el poder estructural juega un papel fundamental a través del papel de las instituciones, sea la CONAGUA y el Ayuntamiento de Morelia, los cuales son importantes en procesos administrativos y legales, pero en la zona de estudio son menos significativos debido a su escaso involucramiento (Figura 2). Otros actores como OOAPAS y los Comités de Agua son partícipes, pero solo se limitan a cuestiones técnicas, sin injerencia en aspectos sociales. Por otro lado, y adicional al poder relacional socio-político que señala Schmidt (2014), se identificó que el mayor peso en el ciclo hidrosocial de La Aldea es a través de actores como Antorcha Campesina y HERSO. Esos actores han tenido repercusiones significativas asociadas a las relaciones que establecen con el municipio, las cuales suelen ser con la finalidad de un beneficio propio y de ventaja respecto a otros actores con poder estructural. Así, las relaciones de poder se orientan sobre la injerencia social y económica, más que la institucional (es permisible), a pesar de que el Estado es quien, ante el marco legal, debería atender el acceso al agua y las formas de ocupación del territorio. 
Figura 2. Diagrama de actores en el acceso al agua en La Aldea



Fuente: Elaborado a partir de las entrevistas realizadas.

\section{Agua de acceso limitado, distribución deficiente y servicio costoso}

El acceso al agua en La Aldea es limitado, principalmente, proviene de cuatro pozos en función, ya que dos fueron cerrados, y uno se encuentra en construcción. En cada pozo se extrae el agua mediante bombas eléctricas, por gravedad es direccionada a pilas (cisternas), y después se instalan ductos para distribuir el agua hacia las viviendas. Con el fin de lograr un acceso equitativo, el servicio fue sectorizado tanto espacial como temporalmente, por lo mismo no se dispone de agua todos los días a excepción de HERSO, siendo que su pila es la más distante (Figura 3). Lo anterior ha derivado en malas interpretaciones y algunos pobladores creen que la transitoriedad del servicio se debe a favoritismos.

Si bien la distribución del agua es por gravedad, el terreno accidentado donde se asientan algunas viviendas representa un problema, especialmente las más alejadas a las pilas, ya que inciden en diferencias de presión y disminución de la cantidad de agua (Figura 4-A); los asentamientos están sobre una pendiente promedio de $8 \%$ excepto Lomas La Aldea y Ampliación La Aldea que se encuentran sobre una pendiente $>15 \%$, (Figura 4-B). 
Lorena García-Estrada - Juan Hernández-Guerrero

Hydrosocial cycle and access to water in the periphery of the city

of Morelia, Mexico: Case study in La Aldea settlement

En ocasiones y ante el desabasto de agua, los habitantes de las zonas más alejadas y pendientes inclinadas suelen optar por la compra de agua a través de pipas (Figura 4-C).

Figura 3. Fuentes de abastecimiento y sectorización del servicio del agua

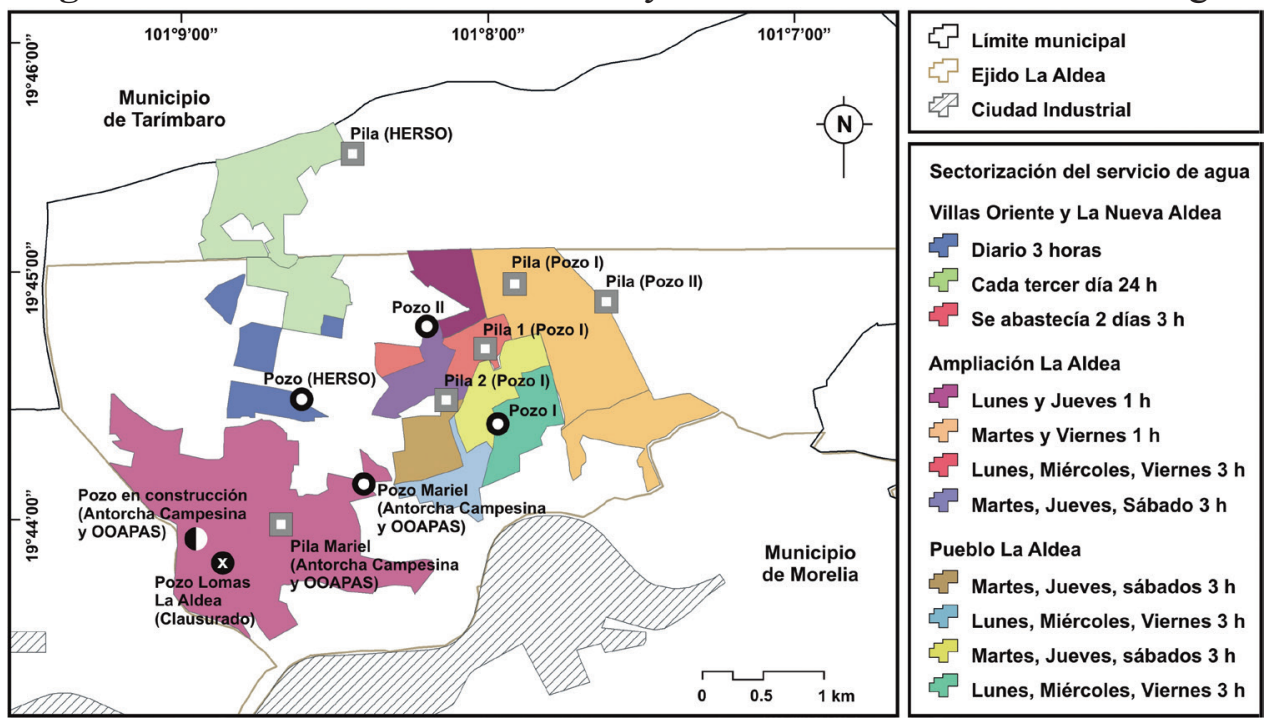

Fuente: Elaboración con datos del INEGI (2010), IMPLAN (2017) y entrevistas.

Figura 4. Condiciones del relieve en la distribución por gravedad del agua en La Aldea

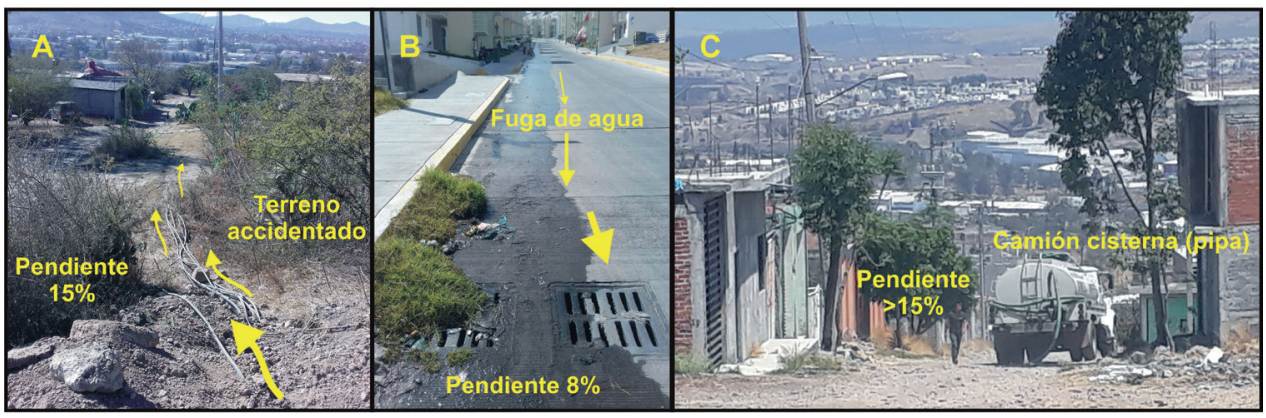

Fuente: Fotografías tomadas por los autores en noviembre 2018. 
Los comités de agua han explicado, que además de las condiciones del terreno, la infraestructura y la llegada de nuevos pobladores son determinantes para que el agua no llegue a la vivienda de forma homogénea. En la figura 5 se muestra la instalación provisional de ductos con materiales frágiles (plástico) (Figura 5-A), conexiones excesivas (cada conexión afecta al resto) (Figura 5-B), válvulas para regular la presión del agua y reparaciones deficientes (Figura 5-C), todo ello forma parte de las estrategias que optan los representantes para solucionar los problemas, pero al utilizar materiales frágiles, provisionales y mal puestos se requiere de reparaciones continuas o reemplazos. Lo anterior propicia que el mantenimiento sea costoso y como respuesta se procura utilizar recursos económicos de la caja de ahorro del comité y/o la cooperación monetaria de la población, pero suele ser insuficiente. En general, la transitoriedad del servicio en suma con las deficiencias de infraestructura provoca que cada vez más personas no cuenten con agua en la vivienda, aunque se destaca que continúa la venta y disposición de conexiones en viviendas de reciente creación y distantes de las pilas.

Figura 5. Infraestructura utilizada en la distribución del agua.

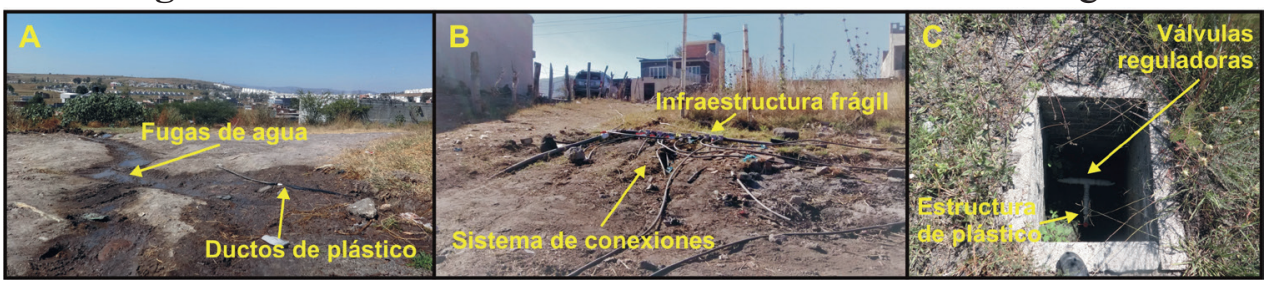

Fuente: Fotografías tomadas por los autores en noviembre 2018.

A pesar de las dificultades, los responsables de brindar la continuidad del servicio de agua tratan de que la población tenga los litros mínimos requeridos para cubrir sus necesidades, pero el discurso que se emplea señala que el agua de los pozos es limitada y la respuesta ha sido sortear temporalmente el suministro de agua. Aun con esa acción, el porcentaje de continuidad es muy bajo en todos los asentamientos, inclusive Villas Oriente que es la más alta con poco más del $50 \%$ del tiempo (Tabla 1). La limitada continuidad no solo incluye el detrimento de la cantidad adecuada de agua a los pobladores, sino que también reduce la vida útil de las instalaciones a través del deterioro de las tuberías. 
Lorena Garcia-Estrada - Juan Hernández-Guerrero

Hydrosocial cycle and access to water in the periphery of the city

of Morelia, Mexico: Case study in La Aldea settlement

Tabla 1. Continuidad del servicio de agua en La Aldea

\begin{tabular}{|l|l|c|c|}
\hline \multicolumn{1}{|c|}{ Asentamiento } & \multicolumn{1}{|c|}{ Servicio } & $\begin{array}{c}\text { Horas a la } \\
\text { semana }\end{array}$ & Continuidad (\%) \\
\hline Pueblo La Aldea & Cada 3er día 3 horas & 10.71 & 6.38 \\
\hline Ampliación la Aldea & 2 y 3 días a la semana 1 o 3 horas & 5 & 2.98 \\
\hline Lomas la Aldea & 2 días a la semana 2 hora & 4 & 2.38 \\
\hline La Nueva Aldea & 7 días 3 horas & 21 & 12.50 \\
\hline Villas Oriente & Cada 3er día 24 horas & 85.71 & 51.02 \\
\hline
\end{tabular}

Fuente: Elaborado con las entrevistas realizadas a los representantes de brindar el agua a los asentamientos.

En lo que respecta a la cantidad de agua, la mayoría de los asentamientos se encuentran por debajo de 150 litros al día por persona (Tabla 2), mientras que la CONAGUA (2015) señala que el consumo promedio de agua por persona en México es de 380 litros al día. Al carecer de información oficial se indagó en la encuesta el consumo de agua aproximado al día (ejemplificado por recipientes de 20 litros), el resultado arrojó un promedio de 140 litros por día (cuando se tiene); equivale a 1 $1 / 2$ recipientes en la ducha (30 litros), 2 en el WC (40 litros), $1 / 2$ en el aseo de manos (10 litros), 2 en el lavado de utensilios para alimento (40 litros) y 1 en el lavado de prendas de vestir (20 litros). Al respecto, la Organización Mundial de la Salud menciona una cantidad aproximada de 20 litros al día por habitante solo para alimentos y aseo personal (CONAGUA, 2015). Ante la insuficiente cantidad de agua para cubrir las necesidades, la respuesta de algunas personas ha sido recurrir a la compra de agua mediante camiones cisterna (conocidas como pipas) o agua embotellada, lo que significa un costo económico extra.

Tabla 2. Cantidad de agua al día por persona en La Aldea

\begin{tabular}{|l|c|c|c|c|c|}
\hline \multicolumn{1}{|c|}{ Asentamiento } & $\begin{array}{c}\text { Número de } \\
\text { habitantes }\end{array}$ & $\begin{array}{c}\text { Número de } \\
\text { habitantes } \\
\text { estimados }\end{array}$ & $\begin{array}{c}\text { Litros en } \\
\text { un día }\end{array}$ & $\begin{array}{c}\text { Litros al día } \\
\text { por persona }\end{array}$ & $\begin{array}{c}\text { Litros al día } \\
\text { por persona }\end{array}$ \\
\hline Pueblo la Aldea & 2522 & 4800 & 1296000 & 513.88 & 270 \\
\hline Ampliación La Aldea & 753 & 1600 & 228571 & 303.55 & 142.85 \\
\hline Lomas la Aldea & 421 & 2000 & 432000 & 410.45 & 86.4 \\
\hline La Nueva Aldea & 578 & 2240 & 172800 & 298.96 & 77.14 \\
\hline Villas Oriente & 8288 & 8288 & 1209600 & 145.95 & 145.95 \\
\hline
\end{tabular}

Fuente: INEGI (2010) y entrevistas a los representantes de brindar agua a los asentamientos. 
En el Pueblo La Aldea y Ampliación La Aldea el costo por el servicio de agua es anual que, junto a la adecuada administración, la mayoría de los habitantes está conforme con el pago por el servicio; Pozo I de $\$ 700$ pesos (US\$36.30) y Pozo II de $\$ 800$ pesos (US\$41.48). Sin embargo, el presidente del comité del Pozo II manifiesta que a pesar del costo, existen vecinos que no realizan el pago, inclusive tienen años con la deuda, situación que limita el presupuesto. Ante dicho problema se intentó retirar el servicio o imponer sanciones, pero los pobladores han sido violentos, de ahí que señalaron como riesgoso el trabajo que desempeña el presidente del comité.

En Lomas La Aldea el costo mensual, hasta el año 2016, ese encontraba entre $\$ 80$ pesos (US\$4.15) y $\$ 120$ pesos (US\$6.22), eso cuando se abastecían del agua del pozo y hasta su cierre (septiembre de ese mismo año). Posterior a ello, se recurre a la compra de agua mediante camiones cisterna con un costo entre $\$ 600$ pesos (US\$31.11) y $\$ 1,200$ pesos (US\$62.22), mientras que aquellos habitantes que se unieron a Antorcha Campesina aseguraron el acceso al agua a través del control de un pozo.

En el caso de La Nueva Aldea y Villas Oriente son los únicos de La Aldea que están exentos del pago por el servicio, eso se debe porque HERSO es la encargada del abastecimiento y en solventar los gastos de servicio hasta que se concluya y entregue el asentamiento al municipio de Morelia (equivale a la legalización del asentamiento), en ese momento será el OOAPAS el responsable de definir precios y cobrar por el servicio.

También es importante considerar el gasto económico para la limpieza de las pilas, reparación de fugas, instalación de válvulas, nuevas conexiones domiciliarias y el pago al bombero. Cada responsable de brindar el servicio recibe un recurso anual con el que realizar el mantenimiento de pozos y pilas, así como reparaciones de fugas, válvulas o nuevas conexiones, aunque el pago mayor se lleva en la energía eléctrica, misma que asciende al año a \$1’374,000 pesos (US\$71,245) (Tabla 3). Al respecto, Lomas La Aldea y Ampliación La Aldea son los asentamientos que presentan mayores complicaciones debido al recurso económico limitado que presentan los habitantes y la deficiente administración. 
Lorena Garcia-Estrada - Juan Hernández-Guerrero

Hydrosocial cycle and access to water in the periphery of the city

of Morelia, Mexico: Case study in La Aldea settlement

Tabla 3. Gasto anual en la administración del acceso al agua

\begin{tabular}{|l|c|c|c|}
\hline \multicolumn{1}{|c|}{ Asentamiento } & $\begin{array}{c}\text { Energía eléctrica } \\
\text { Peso / Dólar }\end{array}$ & $\begin{array}{c}\text { Mantenimiento } \\
\text { Peso / Dólar }\end{array}$ & $\begin{array}{c}\text { Servicio de bombero } \\
\text { Peso /Dólar }\end{array}$ \\
\hline Pueblo de la Aldea & $\$ 390000 /$ US\$20 222 & $\$ 80000 /$ US\$4148 & $\$ 60000 /$ US $\$ 3111$ \\
\hline Ampliación la Aldea & $\$ 540000 /$ US\$28 000 & $\$ 100000 /$ US\$5185 & $\$ 42000 /$ US\$2177 \\
\hline Lomas la Aldea & $\$ 420000 /$ US\$21 778 & $\$ 70000 /$ US\$3630 & $\$ 72000 /$ US\$3733 \\
\hline La Nueva Aldea & $\$ 510000 /$ US\$26444 & - & - \\
\hline Villas Oriente & $\$$ & \\
\hline
\end{tabular}

Fuente: Elaborado con las entrevistas a los representantes de brindar el agua a los asentamientos.

Por otro lado, los principales usos que la población realiza con el agua que recibe es la limpieza de la casa, lavar ropa y utensilios de comida y aseo personal. En el caso de los alimentos, se compra agua embotellada de 20 litros (desde ahora garrafón), ya que no se confía en la calidad del agua que se suministra en la vivienda. Lo anterior implica un gasto adicional, se estima que a la semana el $66 \%$ de la población compra entre 1 y 3 garrafones, un $20 \%$ entre 4 y 6 y un $14 \%$ más de 6 garrafones. Para economizar el gasto se suele acudir a puntos donde se rellenan los garrafones por un precio $\pm \$ 15$ pesos (US\$0.80), eso quiere decir que el gasto puede alcanzar hasta $\$ 90$ pesos (US\$4.7) por 6 garrafones a la semana.

Ahora bien, el $67 \%$ del total de los habitantes del Pueblo La Aldea, Lomas La Aldea y Ampliación La Aldea utilizan el agua pluvial, misma que se capta en cisternas y se utiliza para aseo doméstico y prendas de vestir, pero en el resto de los asentamientos no se considera esa práctica alterna. Aunque cabe decir que el $70 \%$ de los encuestados de todos los asentamientos sí reutilizan agua, especialmente de la ducha o del lavado de prendas de vestir, misma que se usa en el WC o para regar plantas. Cabe decir que ambos casos no son prácticas usuales, pero de implementarse ayudaría en la cantidad de agua y reducir costos.

El desperdicio de agua fue evidente en los recorridos de campo, entre las principales causas destacaron las fugas por los ductos de distribución e infraestructura domiciliaria, el caso más delicado fue Villas Oriente, pues cuatro de los siete días de la semana presentó flujo abundante de agua en las calles e incluso se observaron sitios con humedad.

Con los argumentos anteriores sobresalen dos situaciones. La primera es que el Ayuntamiento de Morelia (2012) reporta que las personas 
de la zona de estudio cuentan con agua independiente de la cantidad, transitoriedad y sectorización del servicio, pero al ser información oficial se integra a los Objetivos de Desarrollo del Milenio y aparece que sí tienen acceso al agua, entonces ¿cuántos lugares presentan y envían reportes bajo estas condiciones? La otra situación es la cantidad de agua embotellada que se compra, sea por la calidad o por su carencia, el fenómeno coincide con cifras a nivel país, ya que el $76.3 \%$ de los hogares en México consume agua embotellada (INEGI, 2018), cifra que se inserta en el rol de poder, economía, necesidad y debilita el ciclo hidrosocial.

\section{Ciclo hidrosocial, cambios en los flujos de agua y las repercusio- nes en la accesibilidad}

Las entradas de agua en La Aldea al año 2012 estaban definidas, en su mayoría, por pozos, seguida de agua embotellada (garrafón de 20 litros) y finalmente, por la obtención de agua a través de camiones cisterna (pipas) (Figura 6). En ese sentido, y aun con los conflictos socio-políticos, el agua de los pozos era suministraba por un servicio eficiente con un costo relativamente bajo, por lo que el agua embotellada se usaba exclusivamente para los alimentos, mientras que la compra de agua por pipas era cuando no se disponía de agua a causa del mantenimiento de pozos y pilas; el promedio mensual por el costo del agua en la vivienda era de $\$ 350$ pesos (US\$18). De lo anterior, la salida del flujo tenía como destino la calle o el drenaje, y era mínimo el re-uso del agua. Adicional, se acentuaron factores que influyeron en el descontrol del acceso al agua, las relaciones sociales comenzaron a debilitarse y los grupos de poder comenzaron a posicionarse en el manejo y gestión del agua. 
Figura 6. Diagrama de flujo del ciclo hidrosocial en La Aldea, 2012.

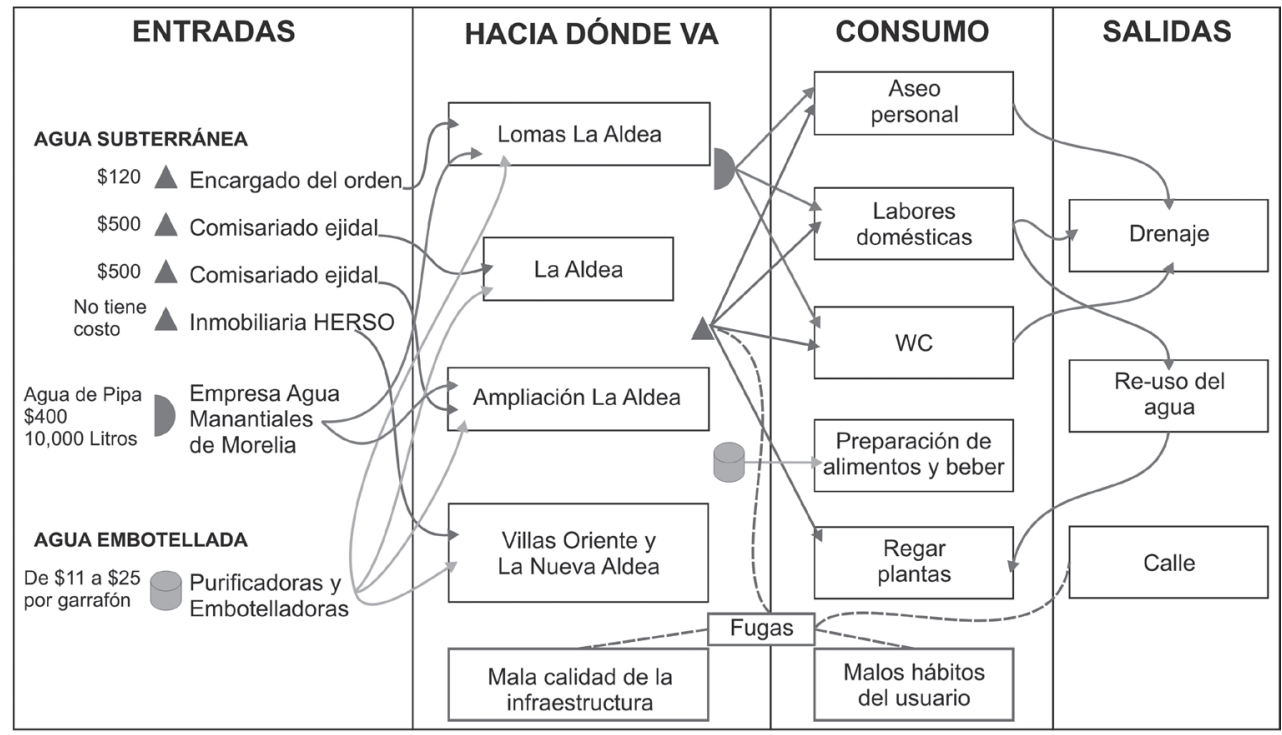

Fuente: Elaboración propia con base en recorridos a campo, encuestas y entrevistas.

Al año 2018, el ciclo hidrosocial evidenció mayores aristas negativas, pues con el crecimiento de los asentamientos el acceso fue menor y el servicio se redujo de horas a días (hasta siete días), aumentaron las inconformidades de la población residente, mientras que los actores con relaciones políticas generaron aún más divisiones, tensiones y fragmentaron el territorio provocando estrés en el ciclo hidrosocial. Así, en la figura 7 se muestra que al año 2018 el ciclo hidrosocial se modificó de forma importante, pues la entrada del flujo de los pozos disminuyó y aumentaron otras formas de acceso al agua (camiones cisterna y agua embotellada) que, hacia el año 2012, eran opcionales, además, el gasto promedio mensual incrementó a $\$ 600$ pesos (US\$31), casi el doble respecto al año 2012, ello propició otras alternativas como el re-uso del agua y la captación de agua de lluvia. 
Figura 7. Diagrama de flujo del ciclo hidrosocial en La Aldea, 2018.

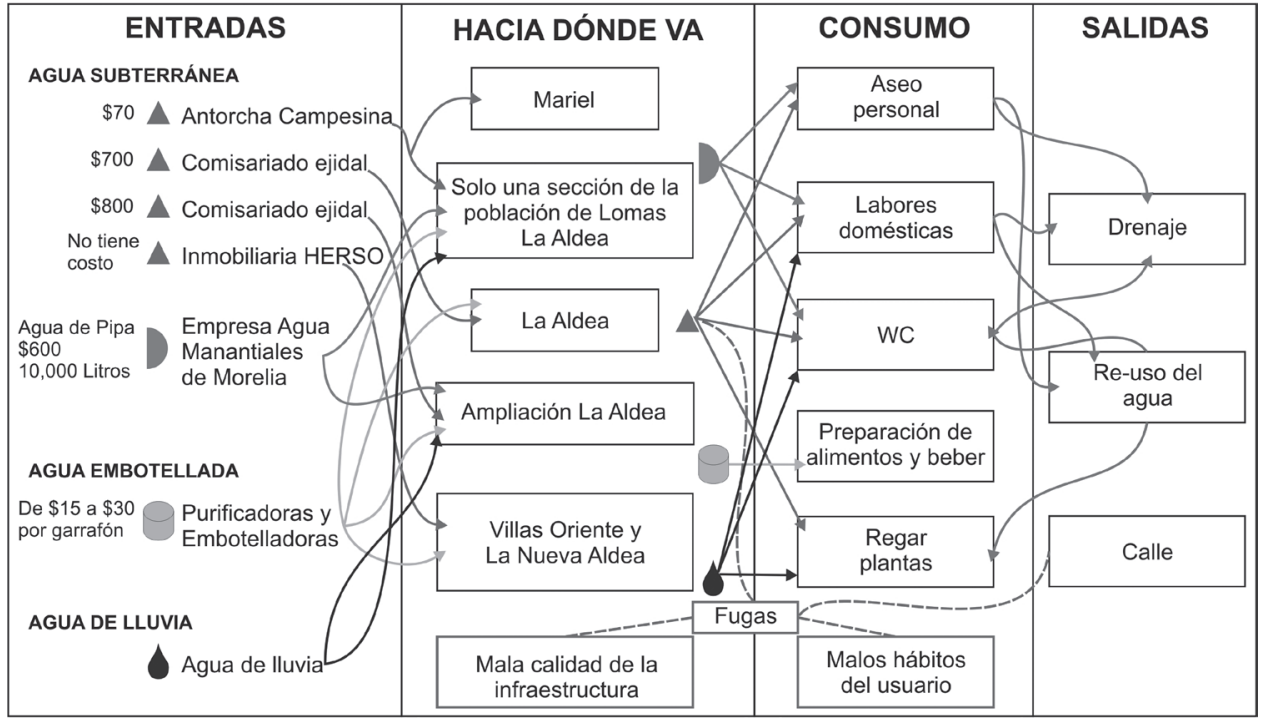

Fuente: Elaboración propia con base en recorridos a campo, encuestas y entrevistas.

Es claro que existe un problema derivado de la insuficiencia de agua subterránea en La Aldea, pero este trabajo deja en evidencia que la mayoría de los problemas son de tipo social y político, entre ellos, el abuso de poder de actores y la escasa atención de las autoridades. Con base en los argumentos de la ecología política que plantea Swyngedouw (2009) y Budds (2012), el presente trabajo ejemplifica el complejo metabolismo entre naturaleza y sociedad, donde se sobresalen las relaciones de poder sobre el control del agua para garantizar intereses individuales y mantener o acrecentar control y dominio. Por otra parte, y en complemento a lo señalado sobre la transformación territorial a partir del agua de Arahuetes et al. (2016), aquí se adiciona la sectorización del propio territorio, tanto física como social, pues todos los asentamientos están conformados en superficies relativamente pequeñas [Villa Oriente (53.9 ha), La Nueva Aldea (19.7 ha), Pueblo La Aldea (70 ha), Lomas La Aldea (125 ha) y Ampliación La Aldea (84.5 ha)] y próximos entre sí, donde la distancia entre tener o no tener agua es de unos cuantos centímetros a metros; todo lo anterior forma un mosaico que influyen en el estrés del ciclo hidrosocial. 
Lorena Garcia-Estrada - Juan Hernández-Guerrero

Hydrosocial cycle and access to water in the periphery of the city

of Morelia, Mexico: Case study in La Aldea settlement

\section{Conclusiones}

Se observó que la periferia urbana de Morelia es un sector poco explorado en temas híbridos del agua y con mínima atención de las autoridades. El enfoque integral del ciclo hidrosocial permitió identificar que el acceso al agua es diferenciado, principalmente por acciones y estrategias de actores con poder social, político y económico. También se pudo distinguir estrés en el ciclo hidrosocial, asociado con indicadores de entradas y salidas de flujo de agua, ya que las entradas son modificadas por intereses socio-políticos, mientras que las salidas se fundamentan en intereses económicos de los afectados. Así, la ciclicidad en el acceso al agua es supeditada por actores con relaciones de poder que gestionan a través de un discurso colectivo, pero con intensiones individualistas, y por otro lado, se encuentra la indiferencia y complacencia de las autoridades por dejar ocupar sitios fuera del reglamento y después no contemplarlos en la dotación de servicios básicos. En suma, se genera una reconfiguración territorial arbitraria producto de las necesidades y posibilidades de los habitantes e intereses de los grupos de poder.

Asimismo, existe poca participación de la población en el tema del agua, provocado por la desmotivación de no ser tomados en cuenta en las decisiones colectivas, por lo que la única respuesta que existe es la de adaptarse y responder de forma espontánea. La respuesta generalizada es la espontaneidad, donde los materiales y estrategias para acceder y distribuir el agua se realizan de forma frágil y rudimentaria. Claramente, es una respuesta ante la necesidad y ausencia del Estado, pero las modificaciones al medio y la cada vez mayor infraestructura deficiente con escasa planeación y proyección, traerá consigo altos costos y conflictos hidrosociales que dificultarán la posterior intervención de la autoridad y la planeación urbana. Por el momento, el OOAPAS sigue realizando de forma esporádica estudios técnicos que se limitan a evaluar la cantidad y calidad del agua en los pozos.

Por otro lado, los procesos de gestión y administración se complejizan conforme la dinámica de los grupos de poder. Todos los asentamientos presentan un grupo de poder, pero entre Antorcha Campesina y el encargado del orden propiciaron la ruptura del tejido social y fomentaron enfrentamientos. Por su parte, el Estado al relegar su labor, nuevamente fortalece a los grupos de poder y quedan desprotegidos el resto de los habitantes, por lo mismo tienen la necesidad de adaptarse o responder con sus recursos para acceder al agua. 
Los hallazgos del trabajo brindan la posibilidad para justificar y establecer bases para políticas públicas enfocadas en generar un verdadero involucramiento de las autoridades a multiescala (federal, estatal y municipal). También, que los pobladores conozcan con mayores argumentos la situación y puedan ser partícipes en la toma de decisiones. Además, ofrece una opción para debilitar a los grupos de poder y trabajar sobre procesos de gestión y manejo del agua de forma colectiva e integral.

Finalmente, ante los problemas derivados en el acceso y distribución de agua, se identificaron iniciativas como el re-uso y captación de agua de lluvia, mismas que pueden contribuir de forma importante en la sostenibilidad hídrica del lugar. Esas actividades han cobrado fuerza en La Aldea, pues su aplicación es acompañada, aunque todavía incipiente, en el aprovechamiento en la diversificación de usos (especialmente en actividades cotidianas) y en la disminución del gasto económico. Es claro que actualmente esas alternativas se llevan a cabo con métodos rudimentarios, pero al eficientizar la infraestructura se esperaría beneficios a una mayor escala. Al mismo tiempo que esas alternativas se consoliden se puede promover, fomentar y sensibilizar el uso adecuado del agua, así como contrarrestar ejercicios de poder e incluir mayor involucramiento de las personas, y por ende, se beneficie y colabore en pro del estrés del ciclo hidrosocial.

\section{Referencias}

Aguilar, G. (2008). Peri-urban, illegal settlements and environmental impact in Mexico City. En: Cities, 25, 133-145. doi.org/10.1016/j. cities.2008.02.003

Arahuetes, A., Villar, R. y Hernández, M. (2016). El ciclo hidrosocial en la ciudad de Torrevieja: retos y nuevas tendencias. En: Revista de Geografia Norte Grande, 65, 109-128. doi.org/10.4067/ S0718-34022016000300006

Ávila, P. (2007). Agua, ciudad y medio ambiente. Una visión histórica de Morelia. Michoacán, México: UNAM/SEDESOL/Ayuntamiento de Morelia

Ayuntamiento de Morelia (2012). Programa Parcial de Desarrollo Urbano de la Zona Oriente de Morelia. Recuperado de: http://conurbamx. com/home/wp-content/uploads/2015/02/Carta-Urbana-de-MoreliaZona-Oriente_comp.pdf. 
Bravo, M., Barrera, G., Mendoza, M., Sáenz, J., Bahena, F., y Sánchez, R. (Eds.). (2012). Contribuciones para el desarrollo sostenible de la cuenca del Lago de Cuitzeo, Michoacán. Michoacán, México: CIGA-UNAM/INIFAP

Budds, J. (2008). "Whose scarcity? The hydrosocial cycle and the changing waterscape of La Ligua river basin, Chile". Contentious geographies: Environment, meaning, scale. Aldershot, UK: Ashgate. (pp. 59-68).

Budds, J. (2012). La demanda, evaluación y asignación del agua en el contexto de escasez: un análisis del ciclo hidrosocial del valle del río La Ligua, Chile. En: Revista de Geografía Norte Grande, 52, 167-184. doi.org/10.4067/S0718-34022012000200010

Budds, J., Linton, J., y McDonnell, R. (2014). The hydrosocial cycle. En: Geoforum, 57, 167-169. doi.org/10.1016/j.geoforum.2014.08.003

Castro, P. y Rodrigues, F. (2017). Cities and water security in the anthropocene: Research challenges and opportunities for international relations. En: Contexto Internacional, 39(3), 521-544. doi.org/10.1590/ S0102-8529.2017390300004

Comisión Nacional del Agua (CONAGUA). (2015). Cuidemos y valoremos el agua que mueve a México. Ciudad de México: CONAGUA/ SEMARNAT.

Delgado-Ramos, G. (2015). Water and the political ecology of urban metabolism: the case of Mexico City. En: Journal of Political Ecology, 22, 98-114. doi.org/10.2458/v22i1.21080

Diario Oficial de la Federación (DOF). (1931). Decreto por el que se expropian superficies de terrenos pertenecientes a los ejidos Cotzio, Santiaguito, Salitrillo, Isaac Arriaga y Colonia Consuelo Alfaro de Vázquez, Tarímbaro y Morelia. México.

Díaz-Caravantes, R. y Wilder, M. (2014). Water, cities and peri-urban communities: Geographies of power in the context of drought in northwest Mexico. En: Water Alternatives, 7(3), 499-417. Recuperado de: http://www.water-alternatives.org/index.php/alldoc/articles/ vol7/v8issue3/261-a7-3-4/file

Domènech, L., March, H., y Saurí, D. (2011). Degrowth initiatives in the urban water sector? A social multi-criteria evaluation of non-conventional water alternatives in Metropolitan Barcelona. En: Journal of Cleaner Production, 38, 1-12. doi.org/10.1016/j.jclepro.2011.09.020 
Domínguez, J. (2006). La gobernanza del agua en México y el reto de la adaptación en zonas urbanas: el caso de la ciudad de México. En: Anuario de espacios urbanos, historia, cultura y diseño, 13, 273296. Recuperado de: http://espaciosurbanos.azc.uam.mx/index.php/ principal/article/view/139/136

Garduño, V., Giordano, N., Ávila, J., Hernández, V., Sámano, A. y Díaz, J. (2014). Estudio hidrogeológico del sistema acuífero de Morelia, Michoacán, para una correcta planificación del territorio. Urbanización, Sociedad y Ambiente. Experiencias en ciudades medias. Morelia, Michoacán: CIGA-UNAM (pp. 197-222).

Gobierno de México (2018). Estrategia Nacional para la puesta en marcha de la Agenda 2030. Objetivos de Desarrollo Sostenible. Recuperado: https://www.gob.mx/agenda2030/documentos/ estrategia-nacional-para-la-puesta-en-marcha-de-la-agenda-2030

Gómez-Valdez, M. y Palerm-Viqueira, J. (2016). El abasto de agua por pipa en el valle de Texcoco, México. En: Tecnología y ciencias del agua, 7(2), 133-148. Recuperado de: http://www.scielo.org.mx/pdf/ tca/v7n2/2007-2422-tca-7-02-00133.pdf

Impulsora de Vivienda de Michoacán S.A. de C.V. (IVEM). (2011). Escritura Pública Número 27992. Morelia, Michoacán.

Instituto Municipal de Planeación de Morelia (IMPLAN). (2017). Sistema de información Geográfica y Estadística de Morelia. Recuperado de: https://implanmorelia.org/virtual/sigem/.

Instituto Nacional de Estadística y Geografía (INEGI). (2010). Censo de población y vivienda 2010. Recuperado de: https://www.inegi.org. $\mathrm{mx} /$ programas/ccpv/2010/default.html

Instituto Nacional de Geografía y Estadística (INEGI). (2018). Módulo de Hogares y Medio Ambiente, comunicado de prensa núm. 262/18, 15 de junio de 2018. Recuperado: http://www.beta.inegi.org.mx/ contenidos/saladeprensa/boletines/2018/

Kallis, G. (2010). Coevolution in water resource development the vicious cycle of water supply and demand in Athens, Greece. En: Ecological Economics, 69, 796-809. doi.org/10.1016/j.ecolecon.2008.07.025

Karpouzoglou, T., Marshall, F., y Mehta, L. (2018). Towards a peri-urban political ecology of water quality decline. En: Land Use Policy, 70, 485-493. doi.org/10.1016/j.landusepol.2017.11.004 
Lorena Garcia-Estrada - Juan Hernández-Guerrero

Hydrosocial cycle and access to water in the periphery of the city

of Morelia, Mexico: Case study in La Aldea settlement

Kaushal, S., McDowell, W., Wollheim, W., Newcomer, T., Mayer, P., Belt, K., y Pennino, M. (2015). Urban evolution: The role of water. En: Water, 7, 4063-4087. doi.org/10.3390/w7084063

Linton, J. y Budds, J. (2014). The hydrosocial cycle: Defining and mobilizing a relational-dialectical approach to water. En: Geoforum, 57, 170-180. doi.org/10.1016/j.geoforum.2013.10.008

López, R. (2013). Sujetos sociales, conflictos y gestión de los servicios de agua potable, alcantarillado y saneamiento en el espacio social-natural de la ciudad de Puebla 1984-2010. (Tesis doctoral. Facultad de Economía. Benemérita Universidad Autónoma de Puebla). México. López-Mares, L., Lozano de Poo, J., Torre-Silva, F., Rodríguez-Santiago, J. y López-Fraga, J. (2019). El ciclo hidro-social de los ríos urbanos: Transformaciones del paisaje hídrico en San Luis Potosí, México. En: Revista de Ciencias Ambientales. Tropical Journal of Environmental Sciences, 53(1), 45-69. doi.org/10.15359/rca.53-1.3

Meerganz von Medeazza, G. (2006). Flujos de agua, flujos de poder. La aportación de Erik Swyngedouw al debate sobre los recursos hídricos en Latinoamérica y en el Estado español. En: Documents d'Anàlisi Geogràfica, 47, 129-139. Recuperado de: https://ddd.uab.cat/pub/ dag/02121573n47/02121573n47p129.pdf

Metha, L., Allouche, J., Nicol, A., y Walnycki, A. (2014). Global environmental justice and the right to water: The case of peri-urban Cochabamba and Delhi. En: Geoforum, 54, 158-166. doi.org/10.1016/j. geoforum.2013.05.014

Morales, M. (2015). Flujos de agua y poder. La gestión del agua urbanizada en la ciudad de Morelia, Michoacán. (Tesis doctoral. Centro de Estudios Antropológicos, El Colegio de Michoacán, A.C.) México. (pp.311).

Naciones Unidas (2010). El derecho humano al agua y saneamiento. Asamblea General del 28 de julio de 2010. Recuperado: http://www. un.org/ga/search/view_doc.asp?symbol=A/RES/64/292\&Lang=S.

Naciones Unidas (2015). Objetivos de Desarrollo del Milenio. [Informe 2015]. Recuperado: http://www.un.org/es/millenniumgoals/

Narain, V. (2016). Peri-urbanization, land use change and water security: A new trigger for water conflicts? En: II Kozhikode Society Management Review, 5(1), 5-7 doi.org/10.1177/227797521561726 
Nieto,C.(2014).LaconstrucciónsimbólicadelmiedoenlaciudaddeMéxico.En: NuevaAntropología,27(81),33-53.Recuperadode:http://www.scielo.org. $\mathrm{mx} /$ scielo.php?script=sci_arttext\&pid=S0185-06362014000200003

Olvera-Molina, M. (2016). Desnaturalizando la cuenca en México: notas sobre el espacio hidropolítico. En: Agua y Territorio, 7, 11-21. doi. org/10.17561/at.v0i7.2959

Rodríguez, A. (2018). Apropiación del agua y configuración hidrosocial en los Altos de Jalisco (1935-2017). En: Secuencia, 101, 167-199. doi. org/10.18234/secuencia.v0i101.1492

Schmidt, S. (2014). Historicising the hydrosocial cycle. En: Water Alternatives, 7 (1), 220-234. Recuperado de: http://www.water-alternatives.org/index.php/alldoc/articles/vol7/v7issue1/242-a7-1-13/file

Secretaría de Desarrollo Agrario, Territorial y Urbano (SEDATU) y Consejo Nacional de Población (CONAPO). (2018). Sistema Urbano Nacional 2018. Ciudad de México: SEGOB/SEDATU/CONAPO.

Secretaría de Medio Ambiente y Recursos Naturales (SEMARNAT) y Comisión Nacional del Agua (CONAGUA). (2015). Atlas del agua en México. Ciudad de México: SEMARNAT y CONAGUA.

Somuano, M. (2007). Movimientos sociales y partidos políticos en América Latina: una relación cambiante y compleja. En: Política y Cultura, 27, 31-53. Recuperado de: http://www.scielo.org.mx/scielo. php?script=sci_arttext\&pid=S0188-77422007000100003

Swyngedouw, E. (1997). Power, nature, and the city. The conquest of water and the political ecology of urbanization in Guayaquil, Ecuador: 1880-1990. En: Environment and Planning A, 29, 311-332. doi. org/10.1068/a290311

Swyngedouw, E. (2009). The political economy and political ecology of the hydro-social cycle. En: Journal of Contemporary Water Research \& Education, 142, 56-60. doi.1111/j.1936-704X.2009.00054.x

Swyngedouw, E., Kaïka, M., y Castro, E. (2002). Urban water: a political-ecology perspective. En: Built Environment, 28(2), 124-137.

Tetreault, D. y McCulligh, C. (2018). Water grabbing via institutionalized corruption in Zacatecas, Mexico. En: Water Alternatives, 11(3), 572-591. 
Vieyra, A., Méndez-Lemus, Y., y Hernández-Guerrero, J. (Coords.). (2018). Procesos periurbanos: desequilibrios territoriales, desigualdades sociales, ambientales y pobreza. Morelia, Michoacán: CIGA-UNAM.

Vilchis-Mata, I., Garrocho-Rangel, C. y Díaz-Delgado, C. (2018). Modelo dinámico adaptativo para la toma de decisiones sostenibles en el ciclo hidrosocial urbano en México. En: Revista de Geografía Norte Grande, 71, 59-90. doi.org/10.4067/S0718-34022018000300059. 
